\title{
Special Issue: Interactive Multimedia Convergence
}

\author{
Jong-Hun Kim ${ }^{1} \cdot$ Raouf Boutaba $^{2} \cdot$ Junseok Yoo $^{3}$
}

Published online: 5 September 2015

(C) Springer Science+Business Media New York 2015

Welcome to the special issue of Multimedia Tools and Applications on "Interactive Multimedia Convergence." This special issue contains a collection of papers that explore techniques, experiences, architectures, and algorithms addressing topics including

- Interactive multimedia computing for convergence

- Multimedia and content protection

- Software infrastructure, middleware, and frameworks for convergence multimedia

- Mobile and wearable computing for convergence information

- Agent technology in convergence information system

- Convergence information applications in u-Commerce, u-Learning, and u-Health

- Innovative application of convergence information

- Cooperative design in convergence environments

- Artificial intelligent methodologies for convergence information

A brief description of each paper follows.

The paper by Lee et al. [1] presents hidden relevant documents buried in larger scientific documents according to terminological paraphrases. The authors propose an effective method of retrieving documents that contain alternative expressions denoting technological concepts in the literature based on predicate argument tuple. The proposed method, which consists of multiple arguments as well as a predicate representing their semantic relationship, expresses both syntactic and semantic interrelations between words in a sentence. As a result, the authors show that PAT-based retrieval could

Jong-Hun Kim

ddcome11@daum.net

Raouf Boutaba

rboutaba@uwaterloo.ca

Junseok Yoo junsyoo@gmail.com

1 Department of Computer Software, Daelim University, Anyang, Republic of Korea

2 School of Computer Science, University of Waterloo, Waterloo, ON, Canada

3 Strategy Department, Autoplatz Corporation, Seoul, Republic of Korea 
identify hidden relevant documents that could not otherwise be retrieved. Therefore, their proposed model can be used as a supplementary model to improve search performance after combining it with other conventional retrieval models. The paper by Chung et al. [6] presents the towards virtualized and automated software performance test architecture. The system architecture considers efficient usage of computer resources, and test automation to reduce human acts is addressed. The paper also describes our proposed method for system architecture and test automation procedures. Using the proposed system architecture, they show how to use the virtual machines, along with which types, for performance measurement. In addition, the six test automation steps are introduced in the paper for the automated testing procedures. Finally, a few experiments were able to show that the proposed schemes may allow automated software performance testing using virtualization.

The paper by Kim et al. [5] introduces a business intelligence application based on the adaptive recognition of user intentions and usage patterns in a mobile environment. This application is named InSciTe Adaptive and is based on text mining as well as semantic Web technologies. InSciTe Adaptive provides several types of technology analyses and prediction services, thereby helping users make better business decisions. Further, it supports technology-focused analysis and predictions, including technology trend analysis, element technology analysis, and convergence technology discovery, as well as adaptive recognition of user intentions by using semiautomatic user modeling processes. The paper by Chung et al. [10] provides a broadcasting method that considers stopping distance and an improved back-off algorithm based on the number of neighbor nodes, which lowers the potential of "chain-reaction collisions," since the vehicle travels at high velocity. This research allows for a broadcasting method for VSC on highways. This method uses a number of neighbor nodes based on how concentrated vehicles are in one area. Rather than sending an emergency message after the collision, faster communication makes predicting traffic hazards possible. Experimenting with NB and IB, the frame reception success rate can be improved.

The paper by Yang [13] introduces a system for building a honeypot farm using a dynamic virtual machine to collect attack information and virtualization technology. The authors propose a dynamic honeypot model consisting of the following components. It is a virtual machine that controls and supervises the state of the virtual machines, an agent that checks IP addresses using the state of the internal domain to create a dynamically virtual machine, a honeypot farm consisting of multiple dynamic virtual machines, a protocolbased intrusion detection system to detect attacks using collected attack information, and a log server to store all records. The paper by Jo et al. [2] presents a social network-based personalized advertisement. The proposed system applies user modeling between collaborative filtering and a frequent pattern network model in order to recommend advertisements according to user conditions. The proposed method alleviates the cold start and sparsity problems of collaborative filtering. Recommendation accuracy will increase when social relations are sufficient.

The paper by Kim et al. [9] presents a facial landmark detection method using an improved active shape model on the Android platform. The authors propose and implement a novel algorithm that extends and enhances the traditional algorithmic state machine (ASM) method except for three modifications. The authors also detail the effects of facial landmarks while implementing an efficient extraction algorithm for facial feature points on 
mobile devices, as most are specific to desktop computers. For this, the authors conducted ASM fitting tests on all frontal images in the face databases. Experimental results revealed that our proposed method is both more accurate and more efficient compared to the classic ASM method. The main contribution of this paper is that the new model can be implemented on the Android platform, which is convenient and can be adapted for use by a large number of applications in the mobile environment. The paper by Park et al. [3] introduces vulnerability of privacy exposure in a mobile data transmission channel. The authors propose a selected major domestic and external website that provides cloud, VoIP, messenger, and e-mail services and inspect whether or not major data are encrypted. The paper also carries out inspection in a wired/wireless environment using Wireshark as an inspection tool. Both wired and wireless environments are verified, and the encryption rate of the ID/password is at an average encryption rate of $85.5 \%$. However, the encryption rate of the data is relatively low at an average encryption rate of $27.8 \%$.

The paper by Lee et al. [7] discusses previous research topics and methods regarding information systems in the fields of supply chain management (SCM) as an analysis of current trends and future directions. The authors were able to identify current trends as well as the most popular research topics and methodologies. One of the most important research methods in empirical research is the lab experiment land survey. The paper further shows that research topics associated with IS in SCM are diversified, whereas research methods are limited due to the lack of theoretical knowledge and qualitative study. The paper by Lee and Song [12] introduces direct optimization of an inference model for human activity and posture class recognition. The authors describe the design and implementation of a class label-tagging system for human activity recognition based on sensor data obtained from a single tri-axial accelerometer attached to the waist of a human subject. Our human activity and posture classifier was designed to more effectively model an event that takes place over a period of time. Consequently, the problem becomes a process of tagging class labels onto sequential data obtained from a single tri-axial accelerometer. The experiment shows that embedding prior probability into the recognition process reduces the error rate in a set of categories. Several experiments proved the efficiency of their proposed method as well as the usefulness of the system.

The paper by Park et al. [11] details performance improvement of an intelligent u-Port system using metallic object applications. For the intelligent u-Port system, the authors manufactured a compact multiple meander antenna to analyze performance deterioration in a metal environment. In the proposed antenna design, the antenna structure consists of a square-shaped power feeder connected to the body for coordination with an attached common-use tag chip, whereas the patch device is manufactured in the form of multiple meanders to efficiently scale back the size of the antenna body. Characteristics of bandwidth, efficiency, and recognition distance were compared and analyzed based on the size of the proposed antenna as well as the number of folds. The results showed that the efficiency and gain characteristics change according to the size of the antenna, and the number of folds in the form of meanders significantly influence the recognition distance of the antenna.

The paper by Kang et al. [4] presents a statistical ontology approach for adaptive object recognition in a situation-variant environment. The proposed method utilizes context ontology, context modeling, context adaptation, and context categorization to design the ontology based on illumination criteria for surveillance. After selecting the 
proper ontology domain, a set of actions is then selected to achieve better performance in that domain. The authors carried out extensive experiments on these concepts in the area of object recognition under dynamic conditions and achieved enormous success that will enable us to proceed with our basic concepts. Consequently, the authors concluded that the performance of the context-aware classifier selection methods for adaptive object recognition is highly dependent upon the environment. The paper by Ko et al. [8] explores how to improve the problems of model verification using verification meta-information and transformation similarity by property matching. In addition, to support verification of the target model generated from the source model, the authors define the verification meta-model for the UML model, RDBMS model, and RT-UML model. By suggesting a verification-based transformation profile for the system structure model, this study provides a new method on how to inspect a converted model, which is one of the most important subjects in model transformation verification research.

This fine collection of papers was achieved by fruitful collaborations. We gratefully acknowledge and express heartfelt thanks to all the authors for their worthy contribution to the special issue. We also would like to thank the reviewers and Professor Borko Furht, editor in chief of the international journal Multimedia Tools and Applications, for his valuable remarks and his undeterred help throughout the publication process of this special issue.

\section{References}

1. Choi S, Myaeng S, Lee D, Lee D (2013) Finding hidden relevant documents buried in scientific documents by terminological paraphrases. Multimed Tools Appl. doi:10.1007/s11042-013-1484-y

2. Ha I, Oh K, Jo G (2013) Personalized advertisement system using social relationship based user modeling. Multimed Tools Appl. doi:10.1007/s11042-013-1691-6

3. Heo GI, Park YJ, Park WH (2013) Vulnerability of information disclosure in data transfer section for constructing a safe smart work infrastructure. Multimed Tools Appl. doi:10.1007/s11042-013-1627-1

4. Kang S, Chung K, Lee J (2013) Ontology-based inference system for adaptive object recognition. Multimed Tools Appl. doi:10.1007/s11042-013-1738-8

5. Kim J, Jeong D, Lee D, Jung H (2013) User-centered innovative technology analysis and prediction application in mobile environment. Multimed Tools Appl. doi:10.1007/s11042-013-1486-9

6. Kim G, Kim Y, Chung K (2013) Towards virtualized and automated software performance test architecture. Multimed Tools Appl. doi:10.1007/s11042-013-1536-3

7. Kim Y, Lee Y, Lee K (2013) An investigation on the information systems research in supply chain management: an analysis of research topic and methodology. Multimed Tools Appl. doi:10.1007/s11042013-1632-4

8. Ko J, Chung K, Han J (2013) Model transformation verification using similarity and graph comparison algorithm. Multimed Tools Appl. doi:10.1007/s11042-013-1581-y

9. Lee Y, Kim CG, Kim Y, Whangbo TK (2013) Facial landmarks detection using improved active shape model on android platform. Multimed Tools Appl. doi:10.1007/s11042-013-1565-y

10. Oh S, Chung K (2013) Traffic hazard prediction based on neighbor nodes for vehicle safety communications on a highway. Multimed Tools Appl. doi:10.1007/s11042-013-1514-9

11. Shin D, Jung H, Lee K, Lee J, Park RC (2013) Performance improvement of intelligent u-Port system using metallic object applications. Multimed Tools Appl. doi:10.1007/s11042-013-1599-1

12. Song M, Lee Y (2013) Direct optimization of inference model for human activity and posture class recognition. Multimed Tools Appl. doi:10.1007/s11042-013-1665-8

13. Yang H (2013) A study on attack information collection using virtualization technology. Multimed Tools Appl. doi:10.1007/s11042-013-1487-8 


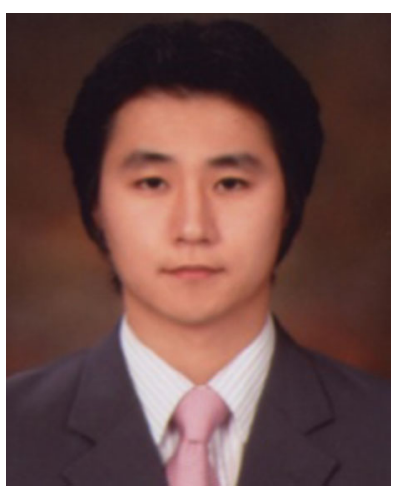

Jong-Hun Kim received a BS degree in physics from the University of Incheon, Korea, in 2001. He received $\mathrm{MS}$ and $\mathrm{PhD}$ degrees in computer science and information engineering from Inha University, Korea, in 2003 and 2010. From 2011 to 2013, he has been manager of U-Healthcare Department, BIT Computer Co., Ltd. He is currently a professor in the Department of Computer Software, Daelim University, Korea. His research interests include data mining, telemedicine, knowledge-based decision support system, intelligent system, multimedia convergence, HCI, and recommendation. He serves as Executive Editing Director of International Conference on IT Convergence and Security (ICITCS), Steering Committees of International Conference on Convergence Technology (ICCT). Also, he is an editorial board member of several international journals.

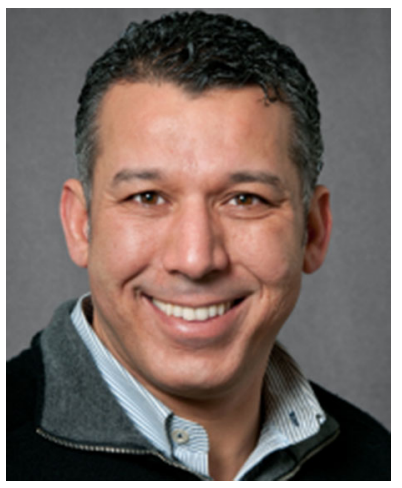

Raouf Boutaba is a professor of computer science at the University of Waterloo and a David Cheriton Faculty Fellow in Canada. He is the founding Editor in Chief of the IEEE Transactions on Network and Service Management (2007-2010), and on the editorial board of several other journals. He served as the general or technical program chair for a number of international conferences. His research interests are in the areas of network and service management. He has published extensively in these areas and received several journal and conference Best Paper Awards such as the IEEE 2008 Fred W. Ellersick Prize Paper Award. He also received several other recognitions such as the Premier's Research Excellence Award, industry research excellence awards, fellowships of the Faculty of Mathematics of the David R. Cheriton School of Computer Science, and outstanding performance awards at the University of Waterloo. He has also received the IEEE Communications Society Hal Sobol Award and the IFIP Silver Core in 2007, the IEEE Communications Society Joe LociCero and the Dan Stokesbury awards in 2009, and the IEEE Communications Society Salah Aidarous award in 2012. He served as a distinguished lecturer for the IEEE Computer and Communications Societies. He is fellow of the IEEE and fellow of the Engineering Institute of Canada. 


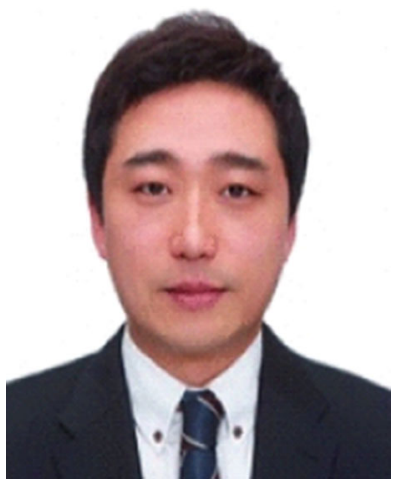

Junseok Yoo is the Deputy General Manager of the Strategy Department at Autoplatz Corporation. He was the executive secretary of the Institute of Creative and Advanced Technologies and Center for Industry Security, Korea. He supervised and managed five conferences including the International Conference on Information Science and Application (ICISA) and the International Conference IT Convergence and Security (ICITCS). His research topics are strategy analysis, automobile, data mining, knowledge, support system, security, HCI, intelligent system, IT convergence, ubiquitous computing, and recommendation. Furthermore, he has controlled 17 special issues of prestigious international journals. Also, he is an editorial board member of several international journals. 